Research Paper

\title{
The suppression of DUSP5 expression correlates with paclitaxel resistance and poor prognosis in basal-like breast cancer
}

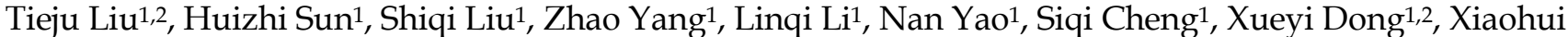 \\ Liang1,2, Chen Chen ${ }^{1}$, Yi Wang ${ }^{1}$, Xiulan Zhao ${ }^{1,2}{ }^{\bowtie}$ \\ 1. Department of Pathology, Tianjin Medical University, Tianjin 300070, China \\ 2. Department of Pathology, General Hospital of Tianjin Medical University, Tianjin 300052, China \\ $\square$ Corresponding author: Xiulan Zhao, Department of Pathology and General Hospital of Tianjin Medical University, Tianjin, China; E-mail: \\ sunbaocun2016@hotmail.com; baocunsun@aliyun.com; Tel:86-13602042200; Fax:86-22-83336813 \\ (c) Ivyspring International Publisher. This is an open access article distributed under the terms of the Creative Commons Attribution (CC BY-NC) license \\ (https://creativecommons.org/licenses/by-nc/4.0/). See http://ivyspring.com/terms for full terms and conditions.
}

Received: 2018.01.16; Accepted: 2018.04.12; Published: 2018.05.16

\begin{abstract}
Basal-like breast cancer (BLBC) is resistant to endocrinotherapy and targeted therapy and new molecular therapies are needed for BLBC. In this study, we evaluated the role of DUSPI and DUSP5, negative regulators of mitogen-activated protein kinase pathway, in the aggressiveness of BLBC. MDA-MB-231 cells were given paclitaxel (PTX) treatment and subsequently PTX resistant cell clones were established. Microarray analysis, real-time quantitative reverse transcription PCR (qRT-PCR), and online analysis of large cohorts of breast cancer patients were performed. The PTX resistant cells showed stronger cell proliferation ability by exhibiting the upregulation of CENPF, CDC6, MCM3, CLSPN and SMCIA expression. Furthermore, DUSPI and DUSP5 expression was significantly downregulated in PTX resistant cells. In addition, in large breast cancer patients' database, both DUSPI and DUSP5 correlated negatively with higher histological grade. DUSPI low expression was obvious in HER2 positive and basal like while DUSP5 low expression was peculiar for basal like compared with other subtypes. Remarkably, low expression of DUSP5, but not DUSPI, was significantly correlated with poor survival of BLBC patients. In conclusion, our data suggest that loss of DUSP5 expression results in PTX resistance and tumor progression, providing a rationale for a therapeutic agent that restores DUSP5 in BLBC.
\end{abstract}

Key words: basal-like breast cancer; DUSP5; paclitaxel resistance

\section{Introduction}

Breast cancer has been considered as heterogeneous disease with different expression of hormone receptors (estrogen receptor, ER, and progesterone receptor, PR) and human epidermal growth factor receptor 2 (HER2)[1, 2]. The basal-like breast cancer (BLBC) is composed of ER-PR-HER2(triple negative) tumors with high expression of basal markers (such as keratins 5, 6, 14, 17, EGF receptor) and proliferation markers[3]. BLBCs approximately occupy $15-25 \%$ of breast cancers. Classically, BLBCs are usually poorly differentiated tumors, with more than $75 \%$ being high grade [4]. They display high mitotic index, dramatic atypia, high nuclear/ cytoplasmic ratio, invading margins, and frequent necrosis [1]. The prognosis of BLBC is usually poorer than that of luminal A (ER+, PR $\geq 20 \%+$, HER2-, Ki67 low expression), luminal B (ER+, PR $<20 \%+$ or Ki67 high expression, HER2-; or ER+, HER2+), and HER2 positive (ER-, PR-, HER2+) subtypes of breast cancer.

Treatment of BLBC has been challenging and the lack of well-defined molecular targets in BLBC renders these tumours insensitive to conventional treatments targeting the hormone receptors or HER2[5]. Given the poor prognosis of BLBC, treatment with chemotherapy is often offered to most patients. The frequent ER-negativity of BLBC as well 
as their high grade with high proliferative index should theoretically confer them sensitivity to chemotherapy, notably to drugs classically used in breast cancer, such as paclitaxel (PTX). However, despite this sensitivity to chemotherapy, BLBC are associated with a relatively poor prognosis: this is the "triple-negative paradox"[1]. Although these cancers may initially respond to original treatment, they become highly resistant to chemotherapy in the metastatic and recurrent disease and thus traditional chemotherapy is still associated with a high risk of relapse and death in a large portion of patients[5-8]. All of these features of BLBC are of particular interest in medicine and it implies that more personalised interventions and the development of tailored treatments for BLBC is urgently needed[9].

Dual-specificity phosphatases (DUSPs) belong to a protein family responsible for dephosphorylating threonine/serine and tyrosine residues on their substrates. DUSPs selectively dephosphorylate the components of the nuclear mitogen-activated protein kinase (MAPK) pathway, and they can either act as classical negative feedback regulators of MAPK pathway or mediate cross talk between different MAPK pathways and between MAPK pathway and other intracellular signal molecules [10-12]. It has been reported that there are currently 25 genes in the DUSPs family designated as DUSPs, namely DUSP1-28, with DUSP17, -20, and -23 redundantly assigned as DUSP19, -18 , and -25 , respectively[13].

It is now clear that individual DUSPs can exhibit either tumour suppressor function or can act as oncogenes and this might be determined by the expression levels of extracellular signal-regulated kinase (ERK) that are either permissive for or provoke cell proliferation or inversely cause cell cycle arrest or cell death[11]. It has been reported that DUSP1 inhibits carcinogenesis in hepatocellular carcinoma and head and neck squamous cell carcinoma as an ERK inhibitor[14]. Recent work reveals a dynamic pattern of DUSP1 expression within the tumor microenvironment and loss of DUSP1 expression is a characteristic of tumor-derived stem cells[15]. Decreased total DUSP1 protein levels may be considered as a poor prognostic factor in breast cancer[16]. Researchers have found that DUSP1 knockdown in sensitive non-small cell lung cancer cells conferred chemotherapy resistance, but DUSP1 gene silencing in vivo significantly heightened response to paclitaxel and increased apoptosis in ovarian cancer[17, 18]. Moreover, there are also reports that DUSP1 could promote carcinogenesis. The increased expression of DUSP1 was found in prostate, colon, bladder, and pancreatic cancer[14]. In such condition, c-Jun $\mathrm{N}$-terminal protein kinases
(JNK) activation would be inhibited following DUSP1 expression increase, which subsequently protects cancer cells from JNK-induced apoptosis.

DUSP5, as one of four related mammalian derivable nuclear DUSPs, has been found to act as a negative feedback factor of Ras/ERK signaling which could determine Ras pathway activity and functions in Ras/ERK-related cancers[11, 19]. Furthermore, the increased expression of DUSP5 in response to growth factor stimulation is ERK dependent and it can inhibit ERK activity by binding inactive ERK in the nucleus. Therefore DUSP5 might act as a tumour suppressor [19]. Loss of DUSP5 expression has been detected in advanced gastric and prostate cancers, and is associated with poor survival. Furthermore, the exogenous expression of DUSP5 in gastric cancer cells inhibited cell proliferation and colony forming ability in vitro[20, 21]. Microarray analysis of gene expression profiling has also demonstrated the reduced expression of DUSP5 in malignant transformation of breast cancer[22]. DUSP5 was specifically upregulated in luminal A MCF-7 cells treated with phorbol 12-myristate 13-acetate, the activator of MAPK phosphorylation, and this upregulation was correlated with the shutdown of ERK pathway[22]. However, the role of DUSP5 in basal-like breast cancer isn't reported so far.

In this study, we found DUSP1 and DUSP5 downregulation in PTX resistant cells of MDA-MB231 using microarray analysis and quantitative realtime polymerase chain reaction (qRT-PCR), which might be responsible for malignant progression and chemotherapy resistance in BLBC. Specifically, DUSP5 downregulation, rather than DUSP1 downregulation, was peculiar characteristic of BLBC and showed close relationship with poor survival of BLBC patients.

\section{Results}

\section{Paclitaxel (PTX) treatment in MDA-MB-231 cells and generation of resistant cell clones}

BLBC cell line MDA-MB-231 was given paclitaxel $(10 \mathrm{nM})$ treatment for 5 days. Then most cells died in 2 weeks (Figure 1A, B). A small number of residual cells survived and established proliferation clones in 3-4 weeks (Figure 1C), and such cells were considered to be PTX resistant cells.

\section{DEG detection, validation, and functional analysis of PTX resistant cell clones by microarray}

Analysis of GeneChip ${ }^{\circledR}$ Human Transcriptome Array (HTA) data was performed using strict statistical methods to detect the differentially expressed genes (DEGs) in PTX resistant MDA-MB- 
231 cells. The analysis identified 695 DEGs, of which 309 (44.5\%) genes were upregulated and 386 (55.5\%) genes were downregulated.

Gene ontology (GO) enrichment analysis of DEGs was carried out to detect the PTX resistancerelated biological process, molecular function, and cellular component. Table S1 showed the top ten GO functions of DEGs regulated in biological process category in PTX resistant MDA-MB-231 cells (listed in the order of significance from highest to lowest): mitotic cell cycle, apoptosis, cell adhesion, DNA replication, cellular nitrogen compound metabolic process, response to drug, angiogenesis, cell cycle checkpoint, nuclear mRNA splicing, via spliceosome, RNA splicing. Intriguingly, PTX resistant MDA-MB231 cell clones exhibited stronger cell proliferation ability. The proliferation markers of malignant cell growth such as CENPF, CDC6, MCM3, CLSPN and SMC1A were identified as significantly upregulated genes in our microarray analysis and further validated by qRT-PCR (Figure 2A). Many of the PTX resistance-related genes were functionally connected into interplay networks, as analyzed by the Search Tool for the Retrieval of Interacting Genes/Proteins (STRING) (Figure 2B). One large group of PTX resistance-related genes was related to mitotic cell cycle and apoptosis. The other group included many genes involved in cell adhesion (Figure 2B). Taken together, these results suggest that the biological processes related to PTX resistance might be involved in cell proliferation, apoptosis and adhesion.

\section{DUSPI and DUSP5 were downregulated in PTX resistant BLBC cell clones}

Among the identified DEGs, the top ten genes (DUSP1, DUSP5, UGCG, CTGF, SAT1 and GPR110 were downregulated; CCL2, HNRNPM, CDH11 and HIST1H1T were upregulated) were selected according to the absolute value of fold change for further qRT-PCR validation (Figure 3A, B). The two members of DUSPs family, DUSP1 and DUSP5 attract our attention because of the deregulated DUSPs expression in cancers and that DUSPs are a desirable target for therapeutic use due to their small size and their simple domain structure[13].

DUSP1 and DUSP5 expression levels were significantly downregulated in PTX resistant MDA-MB-231 cell clones compared with control cells (fold change: $0.17 ; P<0.001$ and fold change: $0.23 ; P<$ 0.001, respectively) (Figure 3A). The other BLBC cell line Hs578T cells also exhibited significant downregulation for DUSP1 (fold change: 0.42; $P<$ 0.001 ) and DUSP5 (fold change: 0.39; $P<0.001$ ) in survival cell clones after PTX treatment by using qRT-PCR (Figure 3C). These findings suggest the importance of declining expression of DUSP1 and DUSP5 in PTX resistance of BLBC.

\section{Validation of the downregulated DUSP5 expression in BLBC patients using ONCOMINE and GOBO databases}

To assess the expression of DUSP1 and DUSP5 in large samples, we analyzed breast cancer data from ONCOMINE database that can categorize the samples to PAM50 subtypes. The expression of DUSP1 was lower in luminal B $(\mathrm{n}=492 ; \mathrm{P}<0.001)$, HER2 $(\mathrm{n}=240$; $P<0.001)$, and basal $(\mathrm{n}=331 ; P<0.001)$ subtypes when compared with luminal A ( $\mathrm{n}=721)$ (Figure 4A), suggesting that the reduced expression of DUSP1 correlated significantly with the molecular subtypes. Luminal A exhibited the highest DUSP1 expression, while the decreasing expression order was observed in luminal B, basal subtype, and HER2 subtype being the lowest (Figure 4A).

Expression of DUSP1 was also assessed by using data from GOBO for 1881 cases of breast cancers[23]. Using the PAM50 subtypes, DUSP1 expression was significantly lower in basal $(n=304)$, HER2 $(n=240)$ and luminal B $(\mathrm{n}=471)$ subtypes compared with luminal A $(\mathrm{n}=465)(P<0.001)$, consistent with ONCOMINE data. However basal subtype showed the lowest expression level of DUSP1 in this cohort (Figure 4C). DUSP1 expression was also correlated negatively with higher histological grade being the lowest in grade 3 cases $(n=239$ for grade $1, n=677$ for grade 2 , and $n=495$ for grade $3, P<0.001$ ) (Figure $4 \mathrm{C})$.

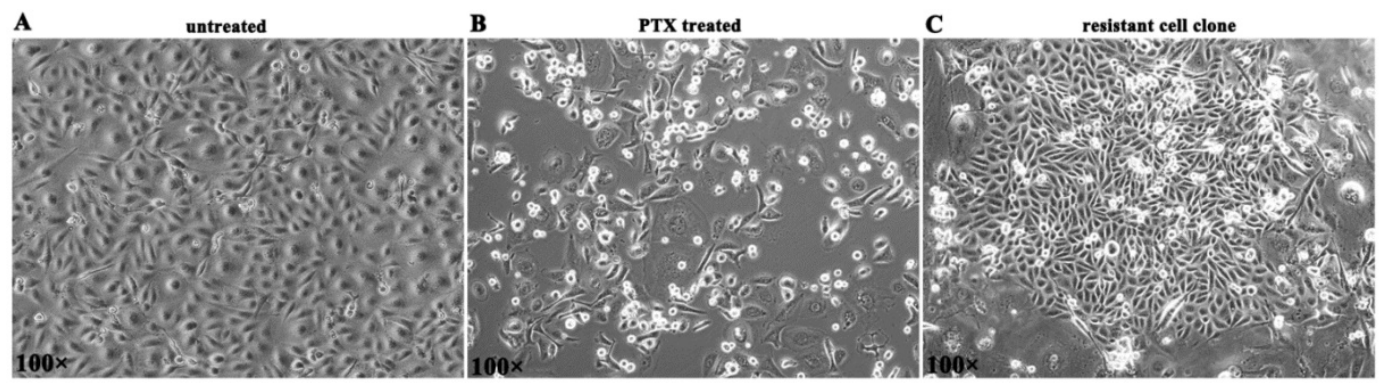

Figure 1. The generation of PTX resistant cell clones. (A) MDA-MB-231 cells in normal culture. (B) Most cells died in 2 weeks after $5 \mathrm{~d}$ of PTX exposure. (C) PTX resistant cell clone was established. 
A

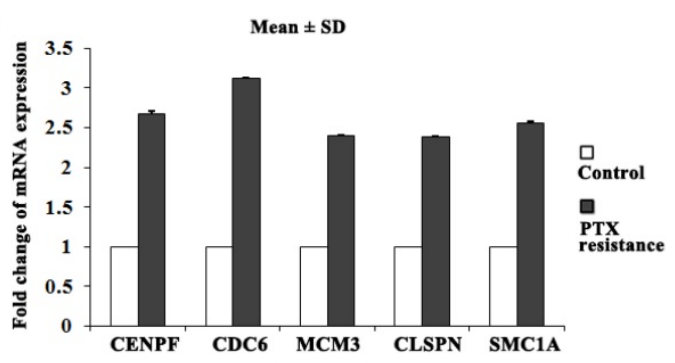

B

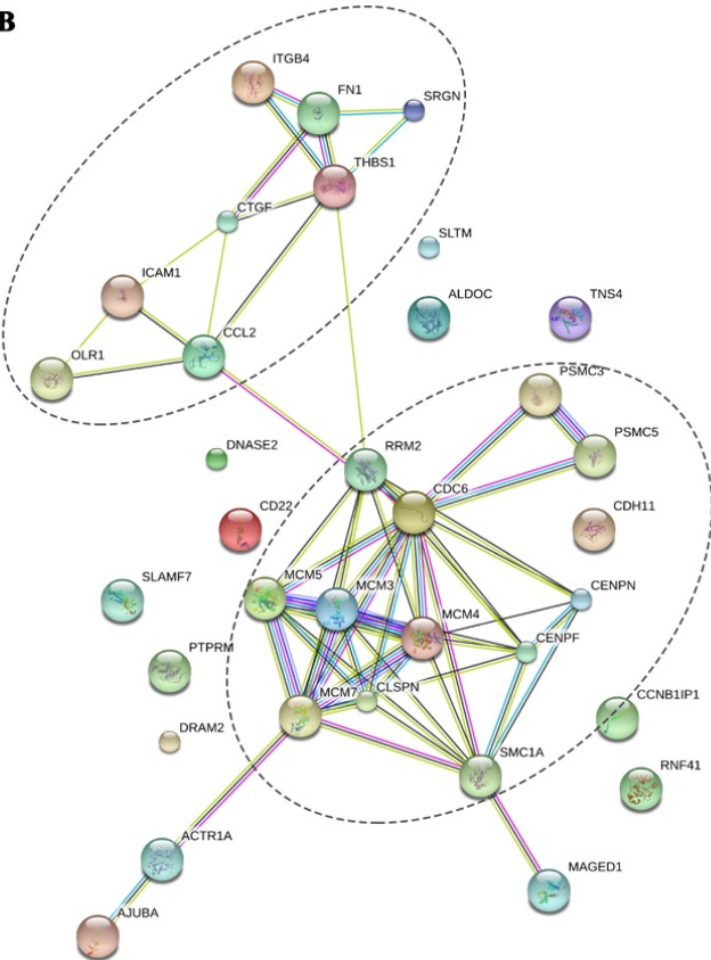

Figure 2. PTX resistance-related genes were functionally connected. (A) CENPF, CDC6, MCM3, CLSPN and SMCIA expression was upregulated in PTX resistant MDA-MB-231 cells by qRT-PCR. (B) The functional association networks of PTX resistance-related genes were analyzed using the STRING database, with subgroups marked by their functions.

Interestingly, the pattern of DUSP5 expression in PAM50 subtypes was different from DUSP1 in ONCOMINE and GOBO data sets. In the cohort of ONCOMINE data, the expression of DUSP5 was slightly higher in luminal $\mathrm{B}(P=0.002)$ and in HER2 $(P$ $=0.044$ ) when compared with luminal $\mathrm{A}$ (Figure 5A, B). However, a noticeable decrease of DUSP5 expression was shown in basal subtype compared with the other three subtypes (Figure 5A, B, P < 0.001).

The identical expression pattern of DUSP5 was also found in GOBO data set using the PAM50 subtypes. DUSP5 expression was significantly reduced in ER-negative $(\mathrm{n}=395)$ tumors compared with ER-positive $(\mathrm{n}=1225, P<0.001)$ (Figure 5C). Compared with luminal A, luminal B and HER2 subtypes, basal subtype showed an obvious decrease in DUSP5 expression (Figure 5D, $P<0.001$ ). These results suggested that the downregulated DUSP5 expression might be peculiar for BLBC patients.
Meantime, DUSP5 expression was also correlated negatively with higher histological grade being the lowest in grade 3 cases (Figure 5E, $P<0.001$ ).

\section{Reduced expression of DUSP5 correlates with poor prognosis in BLBC patients}

In order to analyze the relationship of DUSP1 and DUSP5 expression with survival, Kaplan-Meier (KM) Plotter (www.kmplot.com)[24], which contained gene expression data and survival information of 5143 clinical breast cancer patients downloaded from GEO, EGA and TCGA, was used. To analyze the prognostic value of DUSP1 and DUSP5, patient samples were split into two groups according to lower quartile expression (high vs. low expression) and assessed by a KM survival plot, with the hazard ratio (HR) with 95\% confidence intervals (CI) and logrank $P$ value.

The KM survival analysis showed that although DUSP1 expression wasn't significantly associated with overall survival (OS) of patients with breast cancer (Figure S1A) $(\mathrm{n}=1402, \mathrm{HR}=0.85$ (0.66 - 1.08), logrank $P=0.19)$, it was significantly associated with relapse free survival (RFS) (Figure S1B) $(n=3951$, HR $=0.85(0.75-0.96)$, logrank $\mathrm{P}=0.0075)$. Interstingly, DUSP5 expression was not only associated with OS of patients with breast cancer (Figure S1C) $(n=1402$, HR $=0.76(0.6-0.96)$, logrank $\mathrm{P}=0.021)$, but also significantly associated with RFS (Figure S1D) $(\mathrm{n}=3951$, HR $=0.67(0.59-0.75)$, logrank $\mathrm{P}<0.001)$. The breast cancer patients with lower mRNA levels of DUSP1 or DUSP5 were predicted to have poor RFS while the lower mRNA levels of DUSP5 alone was poor prognostic marker for OS in breast cancer patients.

Next we evaluated the prognostic value of DUSP1 and DUSP5 expression in basal subtype in this cohort of KM database (Figure 6A-D). DUSP1 expression was neither associated with OS $(\mathrm{n}=241$, $\mathrm{HR}=1.29(0.67-2.48)$, logrank $\mathrm{P}=0.44)($ Figure 6A) nor with RFS ( $\mathrm{n}=618, \mathrm{HR}=1.19$ (0.91 - 1.55), logrank $\mathrm{P}=0.21$ ) (Figure 6B). Remarkably, DUSP5 expression was significantly associated with RFS $(\mathrm{n}=618, \mathrm{HR}=$ 0.58 (0.44 - 0.76), logrank $P<0.001$ ) (Figure 6D). The median RFS of BLBC patients with low DUSP5 expression (14.13 months) was shorter than that of patients with high DUSP5 expression (26 months). Moreover, the median OS of BLBC patients with low DUSP5 expression (34.49 months) was much shorter than that of patients with high DUSP5 expression (80.64 months), and this difference almost reached statistically significant effect $(n=241, H R=0.59(0.35$ 1.01), logrank $P=0.053$ ) (Figure $6 \mathrm{C}$ ). These results suggested that the lower mRNA levels of DUSP5, rather than DUSP1, might be poor prognostic marker for BLBC patients and play roles in PTX resistance. 
A

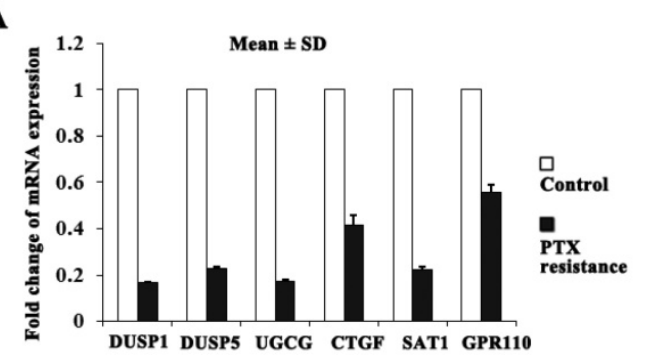

B

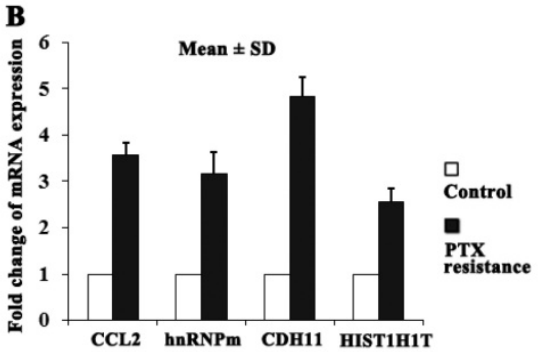

C

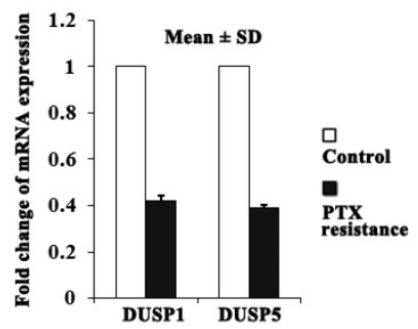

Figure 3. DUSPI and DUSP5 were downregulated in PTX resistant BLBC cell clones. (A-B) The expression of the top ten genes in microarray data was validated by qRT-PCR, and DUSP1 and DUSP5 expression was downregulated in PTX resistant MDA-MB-231 cells compared with control cells. (C) Similarly, DUSP1 and DUSP5 expression was downregulated in PTX resistant Hs578T cells compared with control cells.

$\mathbf{A}$

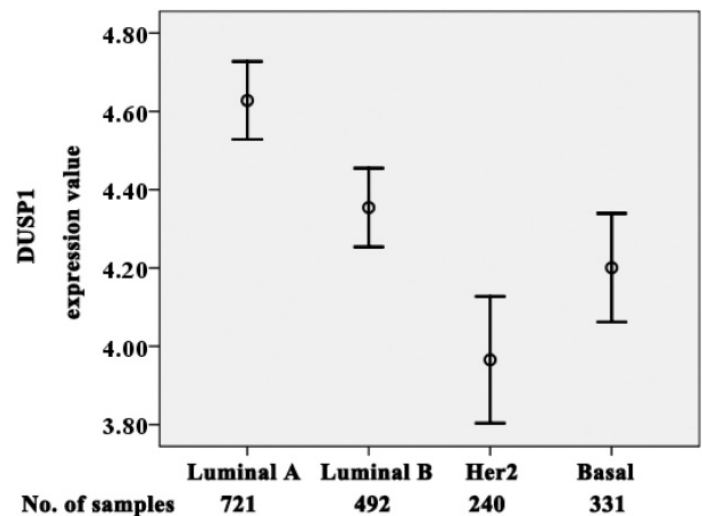

C

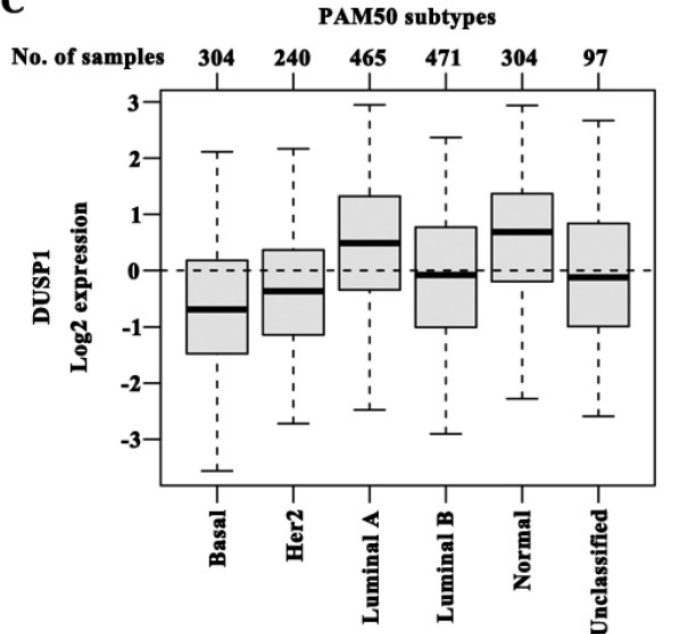

B

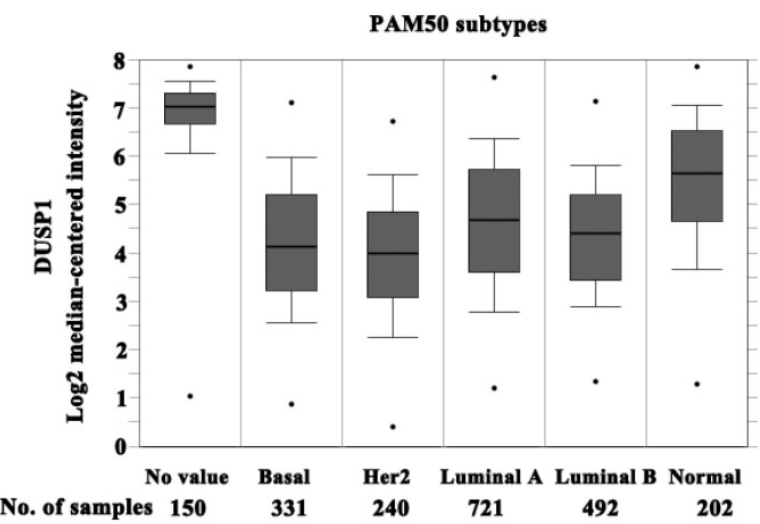

D

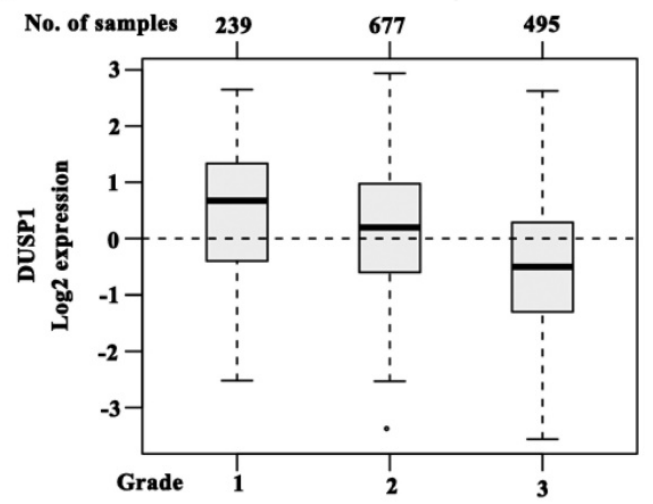

Figure 4. DUSPI expression in BLBC patients. (A-B) The expression of DUSPI was lower in luminal B, HER2 and basal subtypes when compared with luminal A, and HER2 subtype being the lowest by analyzing ONCOMINE data. (C) The expression of DUSPI was lower in luminal B, HER2 and basal subtypes when compared with luminal $A$, and basal subtype being the lowest by analyzing GOBO data. (D) DUSPI expression correlated negatively with higher grade.

\section{Discussion}

BLBC patients usually present with aggressive clinical features, such as metastasis to the lung and brain, high histologic grade and have a poor prognosis and thus need chemotherapy[25]. However, after the chemotherapy, residual cancer cells mostly survive and provoke tumor growth, which contributes to cancer recurrence and mortality.
During the last decades, molecular targeted therapies has been vigorously advocated by precision medicine model [26]. Therefore identifying markers involved in the progression of BLBC would allow the development of targeted therapies.

A growing body of evidence suggests that DUSPs may provide prognostic and predictive utility in several cancers including breast cancer[14, 16]. This 
study demonstrated, for the first time, the mRNA expression and prognostic value of DUSP1 and DUSP5 in basal like breast cancer. Herein, we demonstrated that DUSP1 and DUSP5 expression was significantly downregulated in PTX-resistant BLBC cell lines, suggesting their association with resistance to chemotherapy. Moreover, microarray data, GO analysis and STRING analysis provided evidence that the PTX-resistant BLBC cells was associated with a highly aggressive phenotype, and proliferative markers of malignant cell growth such as CENPF, CDC6, MCM3, CLSPN and SMC1A showed elevated expression in PTX-resistant BLBC cells, suggesting the role of PTX-resistant cells with DUSP1 and DUSP5 downregualtion in BLBC progression.

B

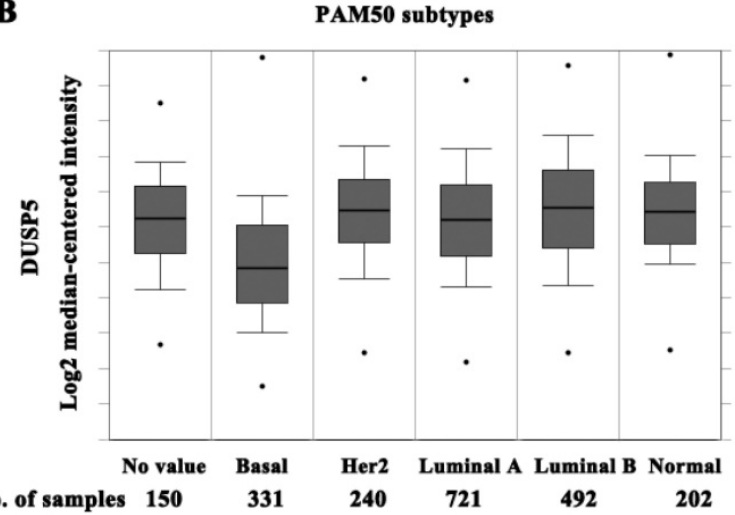

D

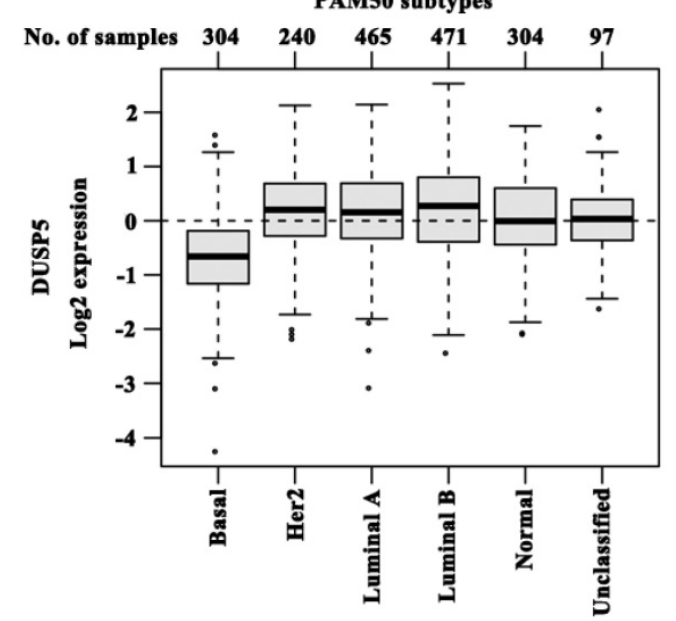

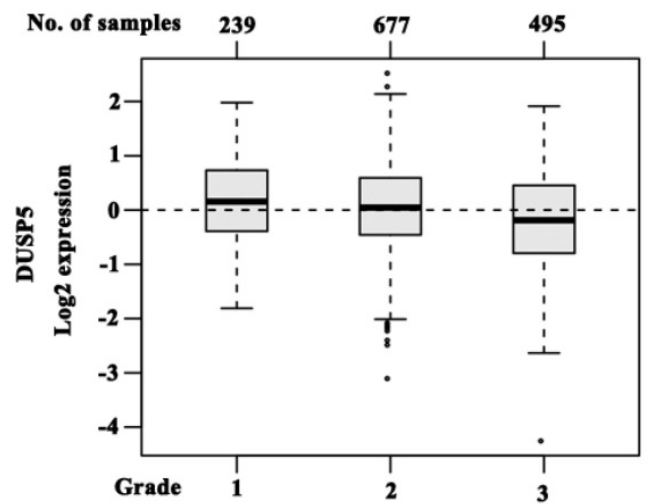

Figure 5. The downregulated DUSP5 expression in BLBC patients. (A-B) Basal subtype showed the lowest expression level of DUSP5 compared with luminal A, luminal $B$ and HER2 subtypes by analyzing ONCOMINE data. (C-D) DUSP5 expression was significantly lower in ER-negative tumors compared with ER-positive (C), and basal subtype showed an obvious decrease in DUSP5 expression compared with luminal A, luminal B and Her2 subtypes (D) by analyzing GOBO data. (E) DUSP5 expression correlated negatively with higher grade. 
A
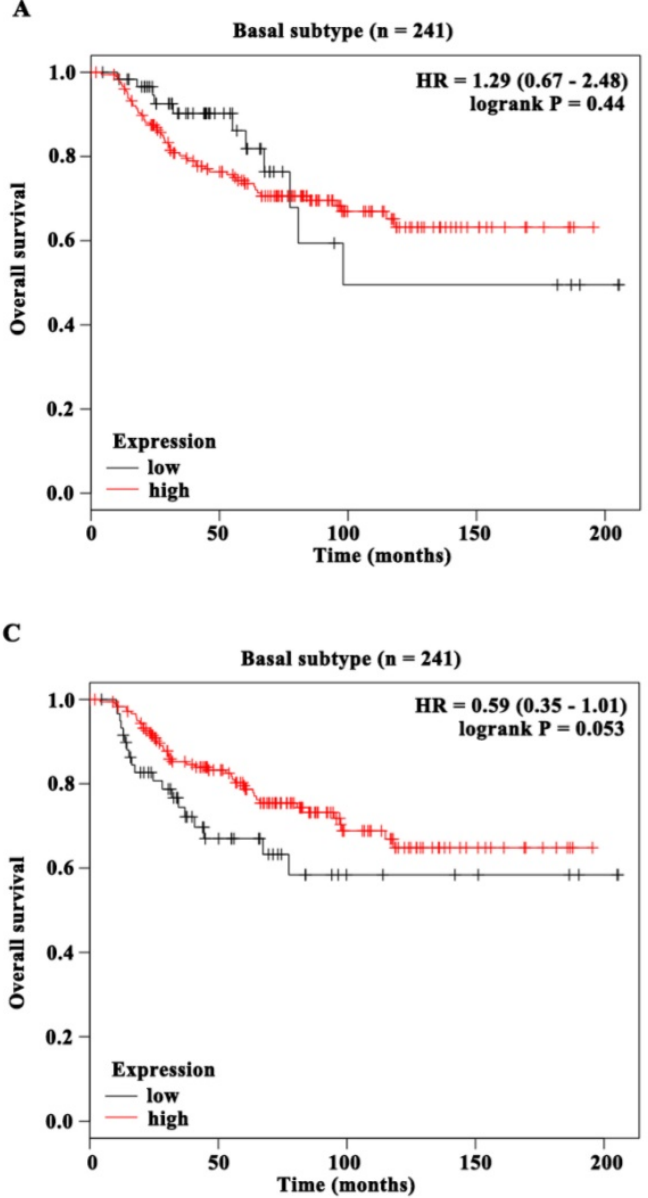

B

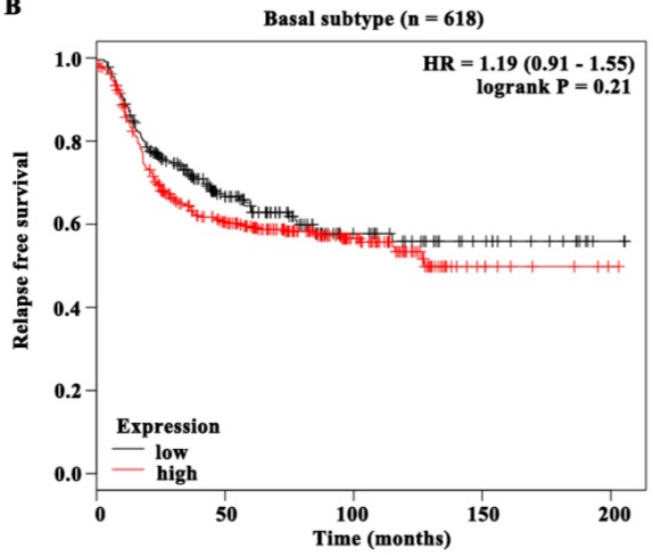

D

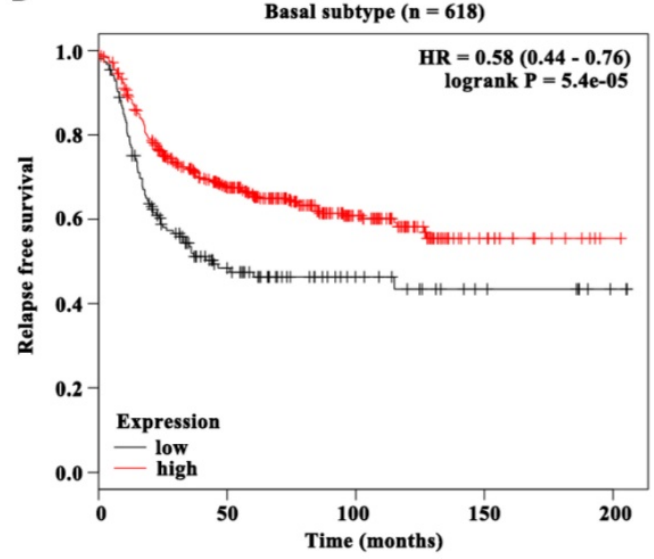

Figure 6. The prognostic value of DUSPI and DUSP5 expression in BLBC patients. (A-B) DUSPI expression was neither associated with OS (A) nor with RFS (B) of BLBC patients. (C) DUSP5 expression was almost significantly associated with OS of BLBC patients and the P value was close to 0.05 . (D) DUSP5 expression was significantly associated with RFS of BLBC patients.

To confirm the data of the cell line studies, we performed analysis in breast cancer patients by using publically available gene expression database. Analyses had been performed using ONCOMINE cohorts, GOBO Affymetrix-based data sets and KM database. These analyses demonstrated the association of low DUSP1 and DUSP5 expression with high histological grade. Although low DUSP1 expression wasn't significantly associated with OS of patients with breast cancer, it was significantly associated with RFS. Low DUSP5 expression was not only associated with OS of patients with breast cancer but also significantly associated with RFS. Therefore these findings together suggested that DUSP1 and DUSP5 functioned as tumor suppressors and might inhibit the progression of breast cancer.

In addition, we found that DUSP1 expression pattern in four subtypes of breast cancer was discrepant in these large publically databases, with it being lowest in HER2 subtype in ONCOMINE database and in basal subtype in GOBO database. These results were consistent with the study of $\mathrm{He} \mathrm{J} \mathrm{et}$ al.[27], which showed that DUSP1 expression was significantly lower in ER-negative breast cancer cell lines (basal like and HER2) than in ER-positive breast cancer cell lines (luminal A and luminal B) by the integrated analysis of GEO database. Taken together, these consistent results suggest that DUSP1 expression may be associated with ER status and could be considered as a potential target gene for the treatment of ER-negative breast cancer.

Importantly, we demonstrated that lower expression of DUSP5 was seen in basal like than in non-basal like breast cancer, with consistent ONCOMINE and GOBO data analysis. DUSP5 expression was lowest in basal like cancers, underlining DUSP5 expression levels were correlated with the basal like subtype of breast cancer and the possible role of DUSP5 in aggressive process of BLBC. Remarkably, further analysis demonstrated the significant correlation of low DUSP5 levels with shorter OS and RFS in BLBC. However, the data revealed that there were no significant differences in OS and RFS between high and low DUSP1 expression. Therefore, it is DUSP5, not DUSP1, may have potential to be a useful biomarker for BLBC and 
additional efforts to explore its clinical significance in BLBC patients are needed in future.

Rushworth LK et al. have demonstrated that DUSP5 is a nonredundant regulator of both nuclear ERK activation and localization, and DUSP5 functions as a tumor suppressor and may play a part in restraining tumor aggressiveness in different cancers[19]. Yan X et al. has found DUSP5 expression is positively correlated with E-cadherin expression, but negatively correlated with $\mathrm{N}$-cadherin and vimentin expression, suggesting it may be involved in regulation of epithelial-to-mesenchymal transition program and tumor progression in advanced colorectal cancer[28]. They also found that high risk stage patients receiving chemotherapy with high DUSP5 expression appeared to have a significantly better survival than those with low DUSP5 expression[28]. The study from Boeckx C et al.[29] also showed chemotherapy resistant cancer cells exhibited low expression of DUSP5 and concomitant ERK signaling activation in head and neck squamous cell carcinoma, suggesting DUSP5 expression as an ERK inhibitor might be a new strategy for overcoming chemotherapy resistance. In our study, the dramatic reduction of DUSP5 expression appeared in PTX-resistant basal like breast cancer cells and basal subtype of breast cancer, suggesting that DUSP5 may participate in various cancer-related biological processes, and loss of DUSP5 expression contributed to drug resistance and tumor progression of BLBC.

In summary, we demonstrate that DUSP5 expression is characteristically downregulated in basal like subtype compared with other subtypes of breast cancer, and may be associated with malignant development of BLBC. We identify DUSP5 expression can serve as a useful prognostic biomarker for BLBC patients. Moreover, we suggest DUSP5 expression is correlated with PTX resistance in basal like cancer cells, which may partly explain its prognostic effect on BLBC patients since most BLBC patients should be given chemotherapy. Overall, these findings collectively demonstrate that DUSP5 has great potential to be translated into clinical practice and induced DUSP5 upregulation could be a promising strategy to overcome PTX acquired resistance in BLBC.

\section{Materials and Methods}

\section{Cell culture and Paclitaxel treatment}

The human breast cancer cell lines MDA-MB-231 were obtained from the American Type Culture Collection. Hs578T cells were provided by the Cell Bank of Type Culture Collection of the Chinese Academy of Sciences, Shanghai, China. These cells were cultured in Dulbecco's Modified Eagle's Medium supplemented with $10 \%$ fetal bovine serum (Hyclone) in a humidified $5 \% \mathrm{CO} 2$ incubator at $37^{\circ} \mathrm{C}$.

Paclitaxel (Selleckchem) treatment for cancer cells was performed as previously described[30]. Briefly, $1 \times 10^{6}$ cells were plated and cultured in $100-\mathrm{mm}$ dishes for $24 \mathrm{~h}$ and then treated with $10 \mathrm{nM}$ paclitaxel for 5 days. Cells were then washed with PBS and maintained in drug-free culture with media replacement every $48 \mathrm{~h}$ until resistant cell clones established.

\section{RNA extraction and microarray analysis}

Total RNA was extracted using Trizol reagent (Tiangen Biotech, Beijing, China), and sent to Oebiotech (Shanghai, China) for Affymetrix GeneChip ${ }^{\circledR}$ Human Transcriptome Array 2.0 analysis. The microarray data have been deposited in NCBI's Gene Expression Omnibus (GEO) (Liu et al., 2016) and are accessible through GEO Series accession number GSE90145 (http://www.ncbi.nlm.nih.gov/geo/quer y/acc.cgi?acc $=$ GSE90145).

\section{QRT-PCR}

QRT-PCR was performed as previously described[30,31]. Briefly, $2 \mu \mathrm{g}$ of total RNA was reverse-transcribed into cDNA using a Reverse Transcription Kit (Takara, RR037A). QRT-PCR analyses were performed with Power SYBR Green (Takara, RR820A) in 7500HT Real-Time PCR System (Applied Biosystems, Foster City, CA). GAPDH internal control was used as an endogenous control, and fold changes were presented by using the $2-\Delta \Delta \mathrm{Ct}$ method using the equation $(\Delta \Delta \mathrm{CT}=(\mathrm{Ct}$ gene of interest - Ct GAPDH) treated sample - (Ct gene of interest - Ct GAPDH) control sample). All qRT-PCR reactions were performed in triplicates. The fold change $>2$ or $<0.5$ was considered as significant.

\section{ONCOMINE analysis}

ONCOMINE gene expression array datasets (www. oncomine.org), an online cancer microarray database[32], was used to analyze the expression levels of DUSP1 and DUSP5 in breast cancers. Breast cancer patients were classified into four different subtypes (luminal A, luminal B, HER2-enriched, or basal) based on the PAM50 signature. The expression level of DUSP1 and DUSP5 in luminal B, HER2enriched, and basal was acquired and compared with luminal A breast cancers by using Students't-test.

\section{GOBO analysis}

DUSP1 and DUSP5 expression levels for 1881 breast cancer patients were analyzed based on molecular subtypes and other clinicopathological parameters (stage, grade, nodal status) by using the 
data sets from the gene expression-based outcome for breast cancer online algorithm (GOBO). Clinical characteristics of individual data sets were described previously[23].

\section{The kaplan-meier plotter}

The prognostic value for survival was evaluated using an online database, Kaplan-Meier Plotter (www.kmplot.com)[24]. Only the JetSet best probe set of DUSP1 and DUSP5 were chosen to obtain Kaplan-Meier plots.

\section{Statistical analysis}

Data analysis was performed with the SPSS16.0 software package (IBM). All P values were two-sided, and statistical significance was measured at the 0.05 level.

\section{Abbreviations}

BLBC, basal-like breast cancer; CI, confidence intervals; DEGs, differentially expressed genes; DUSPs, Dual-specificity phosphatases; ER, estrogen receptor; ERK, extracellular signal-regulated kinase; GEO, Gene Expression Omnibus; GO, gene ontology; GOBO, gene expression-based outcome for breast cancer online algorithm; HER2, human epidermal growth factor receptor 2; HR, hazard ratio; HTA, Human Transcriptome Array; JNK, c-Jun N-terminal protein kinases; KM, Kaplan-Meier; MAPK, mitogenactivated protein kinase; OS, overall survival; $\mathrm{PR}$, progesterone receptor; PTX, paclitaxel; QRT-PCR, quantitative real-time polymerase chain reaction; RFS, relapse free survival; STRING, search tool for the retrieval of interacting genes/proteins.

\section{Supplementary Material}

Figure S1. http://www.medsci.org/v15p0738s1.pdf Table S1. http://www.medsci.org/v15p0738s2.xlsx

\section{Acknowledgment}

This work was partly supported by a grant from The National Natural Science Foundation of China (No. 81672870 to T. Liu and No. 81572872 to X. Zhao), and National Undergraduate Training Program for Innovation and Entrepreneurship (No. 201510062001 to H. Sun).

\section{Competing Interests}

The authors have declared that no competing interest exists.

\section{References}

1. Bertucci F, Finetti P, Birnbaum D. Basal breast cancer: a complex and deadly molecular subtype. Current molecular medicine. 2012; 12: 96-110.

2. Kennecke H, Yerushalmi R, Woods R, Cheang MC, Voduc D, Speers CH, et al. Metastatic behavior of breast cancer subtypes. Journal of clinical oncology : official journal of the American Society of Clinical Oncology. 2010; 28: 3271-7.
3. Rane C, Senapedis W, Baloglu E, Landesman Y, Crochiere M, Das-Gupta S, et al. A novel orally bioavailable compound KPT-9274 inhibits PAK4, and blocks triple negative breast cancer tumor growth. Scientific reports. 2017; 7: 42555.

4. Dai X, Li T, Bai Z, Yang Y, Liu X, Zhan J, et al. Breast cancer intrinsic subtype classification, clinical use and future trends. American journal of cancer research. 2015; 5: 2929-43.

5. Leidy J, Khan A, Kandil D. Basal-like breast cancer: update on clinicopathologic, immunohistochemical, and molecular features. Archives of pathology \& laboratory medicine. 2014; 138: 37-43.

6. Milioli HH, Tishchenko I, Riveros C, Berretta R, Moscato P. Basal-like breast cancer: molecular profiles, clinical features and survival outcomes. BMC medical genomics. 2017; 10: 19.

7. Carey L, Winer E, Viale G, Cameron D, Gianni L. Triple-negative breast cancer: disease entity or title of convenience? Nature reviews Clinical oncology. 2010; 7: 683-92.

8. Sorolla A, Ho D, Wang E, Evans CW, Ormonde CF, Rashwan R, et al. Sensitizing basal-like breast cancer to chemotherapy using nanoparticles conjugated with interference peptide. Nanoscale. 2016; 8: 9343-53.

9. Denkert C, Liedtke C, Tutt A, von Minckwitz G. Molecular alterations in triple-negative breast cancer-the road to new treatment strategies. Lancet. 2017; 389: 2430-42.

10. Caunt CJ, Keyse SM. Dual-specificity MAP kinase phosphatases (MKPs): shaping the outcome of MAP kinase signalling. The FEBS journal. 2013; 280: 489-504

11. Kidger AM, Keyse SM. The regulation of oncogenic Ras/ERK signalling by dual-specificity mitogen activated protein kinase phosphatases (MKPs). Seminars in cell \& developmental biology. 2016; 50: 125-32.

12. Low HB, Zhang $Y$. Regulatory Roles of MAPK Phosphatases in Cancer. Immune network. 2016; 16: 85-98.

13. Huang CY, Tan TH. DUSPs, to MAP kinases and beyond. Cell \& bioscience. 2012; 2: 24.

14. Shen J, Zhang Y, Yu H, Shen B, Liang Y, Jin R, et al. Role of DUSP1/MKP1 in tumorigenesis, tumor progression and therapy. Cancer medicine. 2016; 5: 2061-8.

15. Mills BN, Albert GP, Halterman MW. Expression Profiling of the MAP Kinase Phosphatase Family Reveals a Role for DUSP1 in the Glioblastoma Stem Cell Niche. Cancer microenvironment : official journal of the International Cancer Microenvironment Society. 2017.

16. Hou MF, Chang CW, Chen FM, Wang SN, Yang SF, Chen PH, et al. Decreased total MKP-1 protein levels predict poor prognosis in breast cancer. World journal of surgery. 2012; 36: 1922-32.

17. Kang Y, Nagaraja AS, Armaiz-Pena GN, Dorniak PL, Hu W, Rupaimoole R, et al. Adrenergic Stimulation of DUSP1 Impairs Chemotherapy Response in Ovarian Cancer. Clinical cancer research : an official journal of the American Association for Cancer Research. 2016; 22: 1713-24.

18. Lin YC, Lin YC, Shih JY, Huang WJ, Chao SW, Chang YL, et al. DUSP1 expression induced by HDAC1 inhibition mediates gefitinib sensitivity in non-small cell lung cancers. Clinical cancer research : an official journal of the American Association for Cancer Research. 2015; 21: 428-38.

19. Rushworth LK, Kidger AM, Delavaine L, Stewart G, van Schelven S, Davidson $\mathrm{J}$, et al. Dual-specificity phosphatase 5 regulates nuclear ERK activity and suppresses skin cancer by inhibiting mutant Harvey-Ras (HRasQ61L)-driven SerpinB2 expression. Proceedings of the National Academy of Sciences of the United States of America. 2014; 111: 18267-72.

20. Shin SH, Park SY, Kang GH. Down-regulation of dual-specificity phosphatase 5 in gastric cancer by promoter $\mathrm{CpG}$ island hypermethylation and its potential role in carcinogenesis. The American journal of pathology. 2013; 182: 1275-85.

21. Cai C, Chen JY, Han ZD, He HC, Chen JH, Chen YR, et al. Down-regulation of dual-specificity phosphatase 5 predicts poor prognosis of patients with prostate cancer. International journal of clinical and experimental medicine. 2015; 8: 4186-94

22. Nunes-Xavier CE Tarrega C Cejudo-Marin R, Frijhoff J Sandin A, Ostman A et al. Differential up-regulation of MAP kinase phosphatases MKP3/DUSP6 and DUSP5 by Ets2 and c-Jun converge in the control of the growth arrest versus proliferation response of MCF-7 breast cancer cells to phorbol ester. The Journal of biological chemistry. 2010; 285: 26417-30.

23. Ringner M, Fredlund E, Hakkinen J, Borg A, Staaf J. GOBO: gene expression-based outcome for breast cancer online. PloS one. 2011; 6: e17911.

24. Gyorffy B, Lanczky A, Eklund AC, Denkert C, Budczies J, Li Q et al. An online survival analysis tool to rapidly assess the effect of 22,277 genes on breast cancer prognosis using microarray data of 1,809 patients. Breast cancer research and treatment. 2010; 123: 725-31.

25. Chung $\mathrm{S}$, Jin $\mathrm{Y}, \mathrm{Han} \mathrm{B}, \mathrm{Qu} \mathrm{Y}$, Gao B, Giuliano AE, et al. Identification of EGF-NF-kappaB-FOXC1 signaling axis in basal-like breast cancer. Cell communication and signaling: CCS. 2017; 15: 22.

26. Feng YZ, Zhang OY, Fu MT, Zhang ZF, Wei M, Zhou JY, et al. Low expression of PinX1 is associated with malignant behavior in basal-like breast cancer. Oncology reports. 2017; 38: 109-19.

27. He J, Yang J, Chen W, Wu H, Yuan Z, Wang K, et al. Molecular Features of Triple Negative Breast Cancer: Microarray Evidence and Further Integrated Analysis. PloS one. 2015; 10: e0129842.

28. Yan X, Liu L, Li H, Huang L, Yin M, Pan C, et al. Dual specificity phosphatase 5 is a novel prognostic indicator for patients with advanced colorectal cancer. American journal of cancer research. 2016; 6: 2323-33. 
29. Boeckx C, Op de Beeck K, Wouters A, Deschoolmeester V, Limame R, Zwaenepoel K, et al. Overcoming cetuximab resistance in HNSCC: the role of AURKB and DUSP proteins. Cancer letters. 2014; 354: 365-77.

30. Liu T, Sun H, Zhu D, Dong X, Liu F, Liang X, et al. TRA2A Promoted Paclitaxel Resistance and Tumor Progression in Triple-Negative Breast Cancers via Regulating Alternative Splicing. Molecular cancer therapeutics. 2017; 16: $1377-88$.

31. Sun H, Liu T, Zhu D, Dong X, Liu F, Liang X, et al. HnRNPM and CD44s expression affects tumor aggressiveness and predicts poor prognosis in breast cancer with axillary lymph node metastases. Genes, chromosomes \& cancer. 2017; 56: 598-607.

32. Rhodes DR, Yu J, Shanker K, Deshpande N, Varambally R, Ghosh D, et al. ONCOMINE: a cancer microarray database and integrated data-mining platform. Neoplasia. 2004; 6: 1-6. 